\title{
Significaciones atribuidas por futuras educadoras de párvulos a las TIC incorporadas en su proceso formativo
}

Significances given by future nursery school teachers to the ICT incorporated in their training process

\section{Volumen 18, Número 3 \\ Setiembre-Diciembre}

pp. 1-22

Carolina Flores-Lueg

Carmen Mena-Bastías

Lucía Navarrete-Troncoso

Patricia Arteaga-González

Ana Gajardo-Rodríguez

Revista indizada en REDALYC, $\underline{\text { SCIELO }}$

Revista distribuida en las bases de datos:

LATINDEX, DOAJ, REDIB, IRESIE, CLASE, DIALNET, SHERPA/ROMEO, QUALIS-CAPES, MIAR

Revista registrada en los directorios:

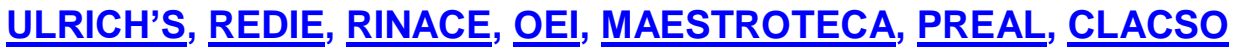




\title{
Significaciones atribuidas por futuras educadoras de párvulos a las TIC incorporadas en su proceso formativo
}

\author{
Significances given by future nursery school teachers to the ICT incorporated in their training \\ process
}

\author{
Carolina Flores-Lueg ${ }^{1}$ \\ Carmen Mena-Bastías ${ }^{2}$ \\ Lucía Navarrete-Troncoso 3 \\ Patricia Arteaga-González ${ }^{4}$ \\ Ana Gajardo-Rodríguez ${ }^{5}$
}

Resumen: Las Tecnologías de la Información y la Comunicación (TIC) y su presencia en la educación, sin duda, han impulsado importantes desafíos para el profesorado, pues este actor se ha visto en la necesidad de desarrollar competencias que le permitan incorporar estas herramientas en sus prácticas docentes con fines pedagógicos, aspecto que debe ser abordado desde su formación inicial. Este artículo expone los resultados de un estudio llevado a cabo en una universidad chilena, cuyo propósito estuvo orientado a determinar el significado que estudiantes de la carrera de Pedagogía en Educación Parvularia le atribuyen a las TIC incorporadas en su proceso formativo, desde una dimensión pedagógica. Se utilizó un enfoque cualitativo, con un diseño de estudio de caso. Se desarrollaron dos grupos focales, donde participaron dieciséis estudiantes, ambos fueron transcritos en forma literal y posteriormente, analizados bajo un proceso de codificación mixta con apoyo del software Atlas.ti v.7. Los resultados evidencian una valoración positiva de estudiantes sobre el uso de TIC con fines pedagógicos en la educación infantil, pero para incorporarlas en el aula primero requieren estar preparadas para ello, aspecto escasamente intencionado dentro del proceso formativo por cuanto solo cursan una asignatura orientada al uso instrumental de estas en primer año de estudios, por lo que sienten una insuficiente preparación para incorporarlas pedagógicamente en el proceso educativo de los párvulos. Se concluye sobre la necesidad de incorporar las TIC trasversalmente en el proceso formativo, con base en experiencias prácticas que permitan una apropiación pedagógica de ellas para transferirlas al aula.

Palabras clave: formación docente, educación infantil, TIC

\begin{abstract}
Information and Communication Technologies (ICT) and its presence in education has undoubtedly given rise to important challenges to teachers, as this actor has have had the need for developing competences that allow them to incorporate these tools into their teaching practices for pedagogical purposes, aspect that must be certainly addressed by the initial training. This article presents the results of a study carried out in a chilean university whose purpose was oriented to establish the significance that students from pedagogy in nursery education attribute to the ICT incorporated in their formative process from a pedagogic perspective. It was used a qualitative approach, a case study design, with a case study design. Data and information collection was carried out through two focus group where sixteen students took part in. Both focus group were transcribed literally and later analyzed by a process of mixed coding supported by Atlas.tiv. 7 software. Results show that students recognize positively the worth of ICT use in children's education, but first, they demand to be prepared to use them, aspect which has been poorly intentioned within the training process, because students only have one subject oriented to the instrumental use of these tools in first year of studies, feeling insufficient preparation to incorporate them pedagogically into the educational process of the preschoolers. It concludes the need to incorporate ICT transversally in the training process, based on practical experiences that allow a pedagogical appropriation of them to transfer them to the classroom.
\end{abstract}

Key words: teacher training, child education, ICT

\footnotetext{
${ }^{1}$ Profesora Universidad del Bío Bío, Chile. Dirección electrónica: cflores@ubiobio.cl

2 Profesora Universidad del Bío Bío, Chile. Dirección electrónica: cmena@ubiobio.cl

${ }^{3}$ Profesora Universidad del Bío Bío, Chile. Dirección electrónica: Inavarrete@ubiobio.cl

${ }^{4}$ Profesora Universidad del Bío Bío, Chile. Dirección electrónica: parteaga@ubiobio.cl

${ }^{5}$ Profesora Universidad del Bío Bío, Chile. Dirección electrónica: agajardo@ubiobio.cl
}

Artículo recibido: 27 de marzo, 2018

Enviado a corrección: 12 de julio, 2018

Aprobado: 13 de agosto, 2018 


\section{Introducción ${ }^{6}$}

Dentro del escenario social actual, caracterizado por la omnipresencia de las Tecnologías de la Información y la Comunicación (TIC), la educación, claramente, adopta una relevancia sin precedentes, pues en ella recae la responsabilidad de formar a la ciudadanía para el siglo XXI, quienes la conforman no solo deben ser capaces de manifestar apertura frente a las nuevas posibilidades que estas herramientas ofrecen y lograr las habilidades necesarias para hacer uso de los nuevos medios tecnológicos/digitales que irán emergiendo, sino que, además, requieren desarrollar aquellas competencias que les permitan aprender durante toda la vida (lifelong learning), y que los prepare para participar activamente dentro de este mundo cada vez más globalizado. Por ello, la competencia digital adquiere cada vez mayor relevancia.

Si se tiene en consideración que la acción educativa es eminentemente una acción social, situada en un tiempo y en un espacio determinado, el proceso formativo de cualquier persona no puede ser comprendido al margen de las características contextuales donde se lleva a cabo. En consecuencia, si las TIC han generado cambios de gran envergadura a nivel de la sociedad, entonces, la escuela y el profesorado se encuentran con el desafío de afrontar estos cambios e incorporar estas herramientas no solo con el fin de optimizar sus procesos administrativos, sino, principalmente, asumirlas como herramientas que pueden ser empleadas como apoyo para la enseñanza y el aprendizaje.

No obstante, diversos estudios evidencian, entre sus resultados, que estas herramientas incorporadas en el aula no han generado el impacto esperado en el aprendizaje del estudiantado ha sido más lento que en otras áreas (González y de Pablos, 2015; Vaillant, 2013). Este fenómeno podría tener su origen en el evidente desfase existente entre la formación que actualmente está entregando la escuela y las exigencias que demanda la sociedad (de Saint Pierre, 2008; Marín-Barbero, 2009; Silva, 2012), lo que sin duda genera ciertos cuestionamientos respecto a la efectividad de las TIC como recurso de apoyo para el proceso de enseñanza y aprendizaje.

La tensión que se genera entre las demandas que presenta la sociedad al sistema educativo y las respuestas que está proporcionando la escuela en torno al mejoramiento de los procesos gracias a la incorporación de las TIC, puede ser explicada a partir de los

\footnotetext{
${ }^{6}$ Este trabajo forma parte del Proyecto de Investigación e Innovación- 2016, fondo interno de la Universidad del Bío-Bío (Chile), titulado "Estrategias de profundización en el uso de TIC desde una perspectiva pedagógica para estudiantes de Pedagogía en Educación Parvularia y su efecto en el nivel de competencia digital" (Código: $160723 / \mathrm{DdoC})$.
} 
modelos epistemológicos que le dan sustento a los programas de formación del profesorado alrededor de estos recursos, pues han sido estructurados bajo una lógica instrumental y no han favorecido una apropiación pedagógica por parte del cuerpo docente (Cabero, Marín y Castaño, 2015; Fainholc, Navia, Romero y Halal, (2013); Unesco, 2004; Vaillant, 2013). Esta situación en gran medida se constituiría en uno de los obstáculos para el proceso de integración de TIC en el aula, porque impide que el profesorado se sienta preparado para innovar en sus metodologías de enseñanza a partir de la incorporación de estas herramientas en sus prácticas pedagógicas.

Al respecto, diversos autores enfatizan en la necesidad de integrar transversalmente estas herramientas en los planes de estudio de la formación inicial docente, por cuanto se constituye en la instancia formativa clave para que las futuras personas docentes adquieran aquellos saberes que les permitan incorporar las TIC en el aula desde una dimensión pedagógica (Gutiérrez y Serrano, 2016; Silva y Astudillo, 2012; Vaillant, 2013). Lo anterior, además, puede ser sustentado por algunas investigaciones que dan cuenta de los insuficientes conocimientos sobre el uso de estas herramientas que presentan estudiantes de pedagogía, donde se destaca que el alumnado no está tan familiarizado con las TIC como se piensa, que ignoran su potencial didáctico y las posibles formas de integración en el currículum de la enseñanza obligatoria (Flores-Lueg y Roig-Vila, 2017; Pérez y Vílchez, 2013).

Por consiguiente, la formación inicial del profesorado en lo que respecta a la implementación y uso de las TIC necesita cambios profundos, requiere de una transformación situada desde las concepciones epistemológicas que subyacen al proceso formativo, pues el solo hecho de incorporarlas en la docencia no necesariamente implica que generen innovaciones metodológicas o que se esté aprovechando todo el potencial educativo que estas herramientas aportan (Flores-Lueg, 2017). Por ello, el desafío principal en la educación no está en transformar los procesos formativos de la formación inicial a partir de la innovación tecnológica, sino con base en la innovación pedagógica que se puede lograr con el apoyo de herramientas tecnológicas.

Por otra parte, reiteradamente, diversas investigaciones destacan que un factor clave para la integración de las TIC en la práctica docente sería la actitud que adopta el profesorado frente a los computadores e Internet, asociada a aspectos cognitivos, afectivos y conativos que determinan el acercamiento hacia estas herramientas y su interacción con ellas en el ámbito personal y profesional (Flores, 2015; Kale y Goh, 2014; Silva y Astudillo, 
2012; Suárez-Rodríguez, Almerich, Díaz-García y Fernández-Piqueras, 2012). Así, se evidencia que factores como el género y el nivel educativo de formación del profesorado también tienen incidencia en el uso pedagógico de estas herramientas en el aula (Barrantes, Casas y Luengo, 2014; Flores-Lueg y Roig-Vila, 2017; Sáinz, 2013; Suárez-Rodríguez et al., 2012), resultados que requieren ser tomados en cuenta si se tiene en consideración la condición de feminización de la carrera docente en los niveles de educación infantil y educación primaria o básica (Sáinz, 2013), porque a raíz de ello, se podría suponer que los niños y niñas que asisten a estos niveles educativos, al estar mayoritariamente bajo la responsabilidad de una profesora, se encontrarían frente a una clara desventaja en cuanto a las formas de interacción con las tecnologías en el aula.

Particularmente, en lo que respecta al proceso formativo de futuras educadoras de párvulos, se han encontrado resultados de investigaciones sobre el desarrollo de algunas experiencias de aprendizaje en las que se ha empleado algún tipo de recurso digital con niños y niñas desde una perspectiva didáctica (Gértrudix y Ballesteros, 2014; Marín-Díaz y Martín-Párraga, 2014), donde se destaca el aporte que estas herramientas realizan al trabajo de los infantes cuando son adecuadamente empleadas en función del aprendizaje. No obstante, las formas de incorporarlas en el aula corresponden a conocimientos particulares y creencias implícitas (Abarzúa y Cerda, 2011), las que también se vinculan a percepciones asociadas a una desvalorización del nivel educativo desde la comunidad escolar y a una desigual distribución de recursos (Espinoza y Rosas, 2016).

Asimismo, se han encontrado estudios sobre el uso de estas herramientas como recursos didácticos en la formación inicial de educadoras de párvulos o maestras de infantil (Cascales, Carrillo y Redondo, 2017; García-Ruiz, Duarte y Guerra, 2014; Hervás-Gómez y Silva-Carmona, 2016), estos se han focalizado en detectar componentes vinculados a la actitud y a la determinación de los niveles de competencia en el uso de TIC. Con ello se evidencia una alta valoración de futuras profesionales en torno a la incorporación de estas herramientas en el aula infantil y un buen dominio de ellas.

En relación con lo anterior y, al tener en cuenta que desde las actuales políticas educativas en Chile se promueve la integración de las TIC en el proceso educativo a partir del nivel de Educación Parvularia y que, además, existe escasa evidencia empírica disponible sobre la efectividad de estas herramientas incorporadas al aula infantil y dentro del proceso formativo de futuras educadoras, surge el interés en realizar este estudio cuyo propósito central se orientó en determinar el significado que estudiantes de la carrera de 
Pedagogía en Educación Parvularia le atribuyen a las TIC incorporadas en su proceso formativo desde una dimensión pedagógica.

\section{Fundamentos teóricos}

\subsection{TIC y formación del profesorado}

Desde hace algún tiempo, y con mayor fuerza hoy en día, existe una creciente preocupación de las instituciones estatales y ONGs dedicadas a la educación respecto a ofrecer e implementar programas de apoyo al profesorado, con el fin de habilitarlos para incorporar las TIC en su labor docente, e idealmente, implementarlas con fines educativos en el aula. No obstante, gran parte de las iniciativas emprendidas han sido estructuradas bajo una lógica técnica o instrumental y no han favorecido una apropiación de estas herramientas desde una perspectiva pedagógica (Cabero et al., 2015; Fainholc et al., 2013; Vaillant, 2013). Si bien el dominio técnico de estas herramientas pareciera ser importante para la práctica docente, no se puede desconocer que el profesorado se encuentra frente al desafío de aceptar que las TIC están a su servicio y que pueden ser aprovechadas como recursos que le permite renovar y/o innovar sus prácticas de enseñanza en función de las nuevas formas de aprender.

En relación con lo anterior, Gutiérrez Marín (2012, p. 50) propone tres dimensiones que se deberían considerar en la formación docente en TIC, a saber:

i) formación como persona y ciudadano del siglo XXI, de la sociedad de la información;

ii) formación didáctica, capacitación como docente y iii) formación como educador, que le haga consciente de cómo los nuevos medios también educan, transforman la sociedad y condicionan su vida y la de sus estudiantes.

Pese a esta propuesta, se considera necesario definir una cuarta dimensión vinculada a la reflexión crítica de la persona educadora, que le permita tomar decisiones sustentadas en aspectos epistemológicos, praxiológicos e ideológicos respecto a decidir incorporarlas en el aula con fines pedagógicos y sobre la forma en cómo las empleará, pues hoy en día se necesita que sea capaz de ejercer una acción pedagógica con ellas (Flores-Lueg, 2015). Del mismo modo, se requiere replantear los modelos a partir de los cuales se están formando los futuros y futuras docentes, por cuanto continúan arraigados a una concepción academicista que no está dando respuesta a las actuales necesidades de los propios estudiantes ni de la sociedad en general (Marín-Barbero, 2009; Vaillant, 2013). 
Lo anterior debe invitar a reflexionar sobre los aspectos ideológicos que sustentan la incorporación de estas herramientas en la educación, a comprender la forma en cómo los profesores y educadoras están construyendo su práctica docente en torno al aprovechamiento de las TIC y cómo se están aproximando al contexto sociocultural vivido por la niñez y la juventud en la actualidad, mediado por estas herramientas.

\subsection{Educación Infantil y TIC}

La educación infantil o parvularia se ha constituido en uno de los niveles formativos que, desde hace algún tiempo, ha estado en el foco de atención de diferentes políticas públicas en Chile y en diversos países latinoamericanos (Bravo y Morales, 2012). Estas políticas tienen su sustento en los aportes teóricos y empíricos emanados desde las neurociencias, los que dan cuenta de la importancia de los primeros años de vida en el desarrollo posterior de un ser humano (Katz, 2005; Ministerio de Educación de Chile (MINEDUC), 2014; Ramírez-Abrahams, Patiño-Mora y Gamboa-Vásquez, 2014), donde el contexto en el cual se desarrollan y las oportunidades que éste ofrezca, para el desarrollo cognitivo, psicomotor y socio-emocional, serían factores claves para el logro de aprendizajes de calidad.

Específicamente, en el marco curricular chileno, vigente para este nivel educativo, se alude a la necesidad de tener en consideración los vertiginosos cambios que están caracterizando a la sociedad, al tiempo que se advierte sobre la necesidad que la educación responda de manera dinámica a los nuevos escenarios que se van configurando y que prepare a las nuevas generaciones para una participación plena acorde con sus posibilidades y sus características. Asimismo, se reconoce que en la actualidad la globalización y revolución de las comunicaciones permiten superar las barreras espacio/temporales para acceder a la información y, en concreto, se señala que estos acontecimientos "abren nuevas posibilidades a los intereses de descubrimiento y de relación de los niños" (MINEDUC, 2001, p. 14). Esto sugiere tener en consideración las posibilidades que ofrecen hoy en día las TIC para acercarlas al proceso educativo de los párvulos, pues permitirían a la persona educadora llevar a cabo una acción educativa contextualizada, pero sin perder de vista la necesidad de rescatar lo propio de cada niño y niña para, a partir de ello, abrir las puertas a otras realidades, a los aportes y oportunidades que ofrece el mundo actual. 
En Chile, una de las primeras apuestas para integrar las TIC al aula de educación parvularia se llevó a cabo en el año 2003 mediante la implementación del proyecto denominado "Kidsmart", con el apoyo de la empresa International Business Machines Corporation (IBM). Este proyecto se implementó en algunas Escuelas de Referencia, con énfasis Tecnológico, denominadas así porque formaron parte de un plan piloto de la implementación de las Bases Curriculares para la Educación Parvularia. Entre los años 2006 y 2007 continúa vigente la política de informática educativa para el nivel, de forma similar a lo iniciado en los años anteriores.

Actualmente, una de las líneas impulsadas para la reforma educativa chilena se relaciona con asegurar una educación de calidad integral y promover la innovación pedagógica para todos los actores involucrados, para ello se propone llevar a las aulas más y mejores tecnologías para el aprendizaje. Particularmente, para el nivel de la educación infantil, a partir del año 2015, desde el Ministerio de Educación chileno, se comenzó a implementar el Proyecto Tablet para Educación Inicial, específicamente para los niveles Transición 1, Transición $2^{7}$ y $1^{\circ}$ Básico, cuyos propósitos fueron favorecer la inclusión y la equidad por medio del acceso de las niñas y los niños a las tecnologías de la información; apoyar a Educadoras y docentes en el aprendizaje de la matemática por medio de un trabajo colaborativo entre pares, centrado en repensar los diferentes ritmos y habilidades de aprendizaje. Cabe destacar que esta iniciativa, hasta el año 2016, considera mil (1.000) establecimientos educativos ubicados en las regiones IV, V, VI, VII, VIII y IX del país (MINEDUC, 2015).

En síntesis, las políticas, en lo que respecta a las TIC incorporadas a la educación infantil a nivel nacional, son escasas, y, al menos en la literatura, no ha sido posible encontrar documentos que den cuenta de los mecanismos implementados para monitorear el aporte de estas al proceso de renovación y/o innovación de las metodologías de enseñanza, ni menos, sobre su contribución real al anhelado mejoramiento de la calidad de los aprendizajes de los niños y las niñas que asisten a la educación formal.

\footnotetext{
7 La Educación Parvularia en Chile corresponde al nivel educativo que atiende a niños y niñas desde su nacimiento hasta los 6 años de edad o su ingreso a la educación básica. Se subdivide en tres niveles educativos: Nivel Sala Cuna, que atiende a niños y niñas entre el nacimiento y los 2 años. El Nivel Medio, que recibe a párvulos desde los 2 a los 4 años; y el Nivel Transición, correspondiente a niños y niñas de entre los 4 y 6 años. Este nivel, a su vez, se subdivide en Nivel Transición 1 (Pre-Kinder o NT1) destinado a párvulos entre los 4 y 5 años; y el Nivel Transición 2 (Kinder o NT2), que recibe a niños y niñas entre los 5 y 6 años de edad.
} 


\section{Metodología}

Este estudio se llevó a cabo bajo el paradigma interpretativo, pues se buscaba lograr una aproximación al mundo personal de los sujetos observando cómo interpretan las situaciones, qué significados les atribuyen y qué intenciones tienen (Touriñán y Sáez, 2012, p. 103). Por consiguiente, desde el punto de vista metodológico, se optó por una investigación cualitativa, centrada en un estudio de caso, entendido éste como un proceso de indagación caracterizado por el examen sistemático y en profundidad de casos de un fenómeno, tales como entidades sociales o entidades educativas únicas (Bisquerra, 2014, p. 309). Como diseño metodológico, se han considerado algunos elementos de la Teoría Fundamentada (Glaser y Strauss, 1967), definida como una "aproximación inductiva en la cual la inmersión en los datos sirve de punto de partida del desarrollo de una teoría sobre un fenómeno" (citado en Guillemette, 2006, p. 32). Además, se ha tenido en cuenta lo planteado por San Martín (2014), cuando expone que "la complejidad del escenario investigativo en el contexto educativo, desafía a construir conocimiento situado desde los sujetos y su mundo de vida" (p. 107). Por tanto, la perspectiva de estudio es de carácter constructivista.

El contexto de estudio correspondió a la carrera de Pedagogía en Educación Parvularia, perteneciente a la Facultad de Educación y Humanidades de una universidad pública y estatal ubicada en la zona centro sur de Chile. Participaron un total de 16 estudiantes, todas mujeres, seleccionadas intencionalmente a partir de los siguientes criterios de inclusión: estar matriculada en la carrera de Pedagogía en Educación Parvularia, en segundo o tercer año de formación en el año 2017 —haber aprobado la asignatura de Tecnología y Aprendizaje - voluntad para participar en el estudio y firmar un consentimiento informado.

Como técnica de obtención de información se empleó el focus group, porque permite acercarse a los significados de los participantes en forma grupal a partir de la generación de conversaciones y debates (De Miguel, 2005, citado en Quintanal y García, 2012, p. 119). Previo al desarrollo de cada focus group, se definieron trece preguntas orientadoras para guiar el diálogo, teniendo en consideración dos dimensiones centrales: i) Valoración de las TIC en educación infantil, en su vida personal y en su proceso formativo; y ii) Nivel de desempeño autopercibido en el uso de TIC. Se llevaron a cabo dos focus group, donde participaron siete estudiantes de segundo año y nueve de tercer año. Cada grupo tuvo una duración aproximada de 40 minutos, fue grabado en audio, previa autorización de las participantes, y posteriormente transcrito en forma literal. 
Cabe destacar, además, que en todo momento se resguardó la identidad de quienes formaron parte de este estudio. Los documentos generados fueron analizados empleando un proceso de codificación mixta (deductiva e inductiva), con apoyo del software Atlas.ti, v.7. Las categorías de análisis finales, de carácter teóricas, fueron definidas teniendo en consideración; por una parte, aquellas dimensiones establecidas en forma apriorística, conforme al propósito de la investigación; y por otra parte, la fundamentación que tuvieron los códigos al momento de hacer los análisis. Es así como se consideraron las siguientes tres categorías centrales: valoración de las TIC en educación infantil $\{11-3\}^{8} ;$ TIC en su vida estudiantil $\{9-6\}$ y desempeño en el uso de TIC $\{10-4\}$, además, se levantaron códigos a partir del discurso de las estudiantes (ver Figura 1).

Figura 1

Red de categorías teóricas y códigos empleados para el análisis de la información proporcionada por estudiantes de Pedagogía en Educación Parvularia

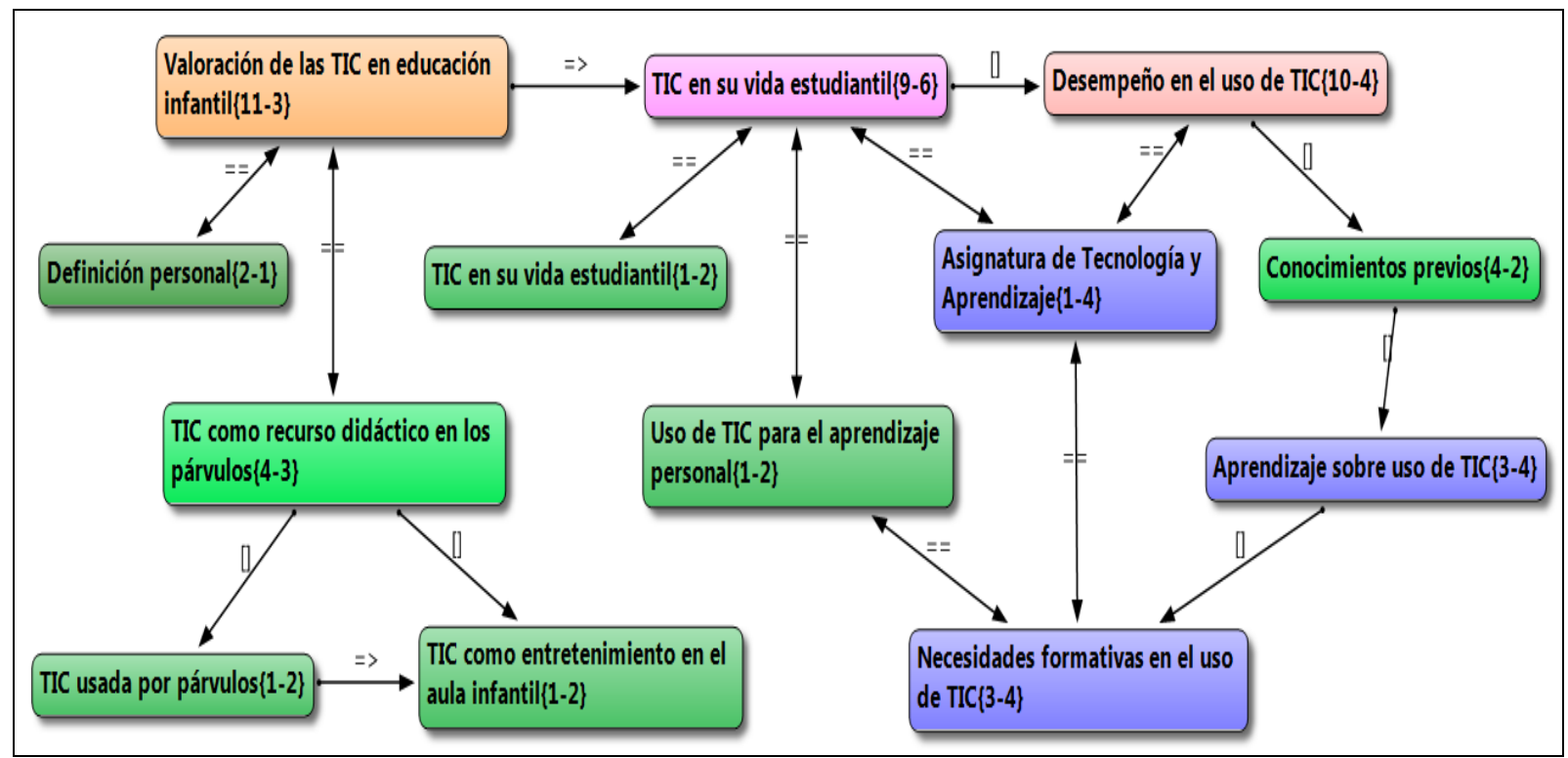

Fuente: Elaboración propia de las autoras con empleo de Atlas.ti. v7

\section{Resultados y sus análisis}

Los resultados se presentan según las tres categorías definidas a partir del análisis de discursos de las estudiantes que participaron en el estudio, vale decir: valoración de las TIC en educación infantil, TIC en su vida estudiantil y desempeño en el uso de TIC.

\footnotetext{
${ }^{8}$ El número de la izquierda representa la "fundamentación" del código, entendida como la cantidad de citas que están vinculadas y que permiten la interpretación; y el número de la derecha se refiere a la "densidad", vale decir, la cantidad de códigos con los que se relaciona el código señalado.
} 


\subsection{Valoración de las TIC en educación infantil}

Las estudiantes señalan que los niños y niñas nacen junto a la tecnología, no obstante, se observa una valoración más bien negativa respecto a la incorporación de estas herramientas a la educación infantil, pero específicamente en lo que respecta al uso que la familia les estaría otorgando, porque perciben que en el hogar las TIC serían utilizadas fundamentalmente para entretener a los niños y niñas. Este sentido otorgado a la tecnología, al decir de las estudiantes, entorpecería el proceso de socialización de los párvulos y tendría repercusiones en el resto de los aprendizajes. Esta valoración puede ser evidenciada cuando señalan lo siguiente:

Son herramientas y un apoyo porque los niños de ahora nacen con estas herramientas, también es una desventaja para el nivel en el que nosotras trabajamos porque los niños llegan a los niveles de sala cuna mayor o menor, lo único que hacen en sus casas es estar con el teléfono o las Tablet o mirando el televisor todo el día donde les proyectan vídeos, canciones, cosas (E1).

Los retrasa porque el niño llega con menos herramientas para socializar con sus compañeros, para acatar órdenes, por así decir que le da la educadora, el niño tiene un desarrollo más lento, no explota las áreas de su cerebro donde él puede conocer por medio de la experimentación, el tacto, entonces eso lo retrasa en cierto modo (E1).

A pesar de las desventajas señaladas respecto al uso de las TIC en el hogar, se reconoce que es necesario incorporarlas dentro de la labor pedagógica, por cuanto corresponderían a herramientas que pueden apoyar el proceso de aprendizaje de los párvulos:

La tecnología tiene un lado bueno y malo, pero igual nos ayuda también para mostrarle a los niños el acceso a las otras culturas, a que puedan tener otro material audio visual, que le podamos ofrecer audios [...] igual en cierto modo hay que saberlas utilizar como profesional, como educadora (E4).

En el fondo las tecnologías son una gran herramienta de apoyo a la educadora de párvulos en general y encuentro que el niño en sí va viendo distintas formas en la que él puede aprender, en la que él puede ir adquiriendo información (E8). 
Principalmente son un apoyo, porque en la sociedad actual es principal esta tecnología, no sé, la sociedad de ahora está sustentada por la tecnología, los niños aprenden con ella, nacen con teléfonos al lado. Deben ser un apoyo y herramienta para nosotras (E10).

Porque es una herramienta de apoyo, que si se sabe ocupar bien, es muy importante y provechosa para el aprendizaje de los niños, y para entregarlo de una manera más lúdica (E9).

Por otra parte, consideran que la incorporación de las TIC en la labor pedagógica se transforma en un importante desafío para las Educadoras de Párvulos, pero destacan que para poder emplearlas en su práctica docente requieren primero tener cierta preparación que las habilite para ello. Esta apreciación puede ser evidenciada cuando señalan lo siguiente:

Existen problemas, desafíos para la educadora que llevan años en el sistema porque ya se lo están pidiendo, no saben cómo utilizarlo, que el niño sepa más que ella puede ser complejo, igual te ayuda, es más complicado para ella el uso porque nunca ha habido (E1).

Tenemos que acercarnos a la tecnología, se usan ahora, es muy actual, entonces no podemos quedarnos atrás con los otros aprendizajes. Tenemos que estar bien preparadas y ayudar a los niños porque posteriormente lo van a seguir utilizando (E6).

Es que como vamos avanzando, los niños van avanzando y como que nosotros tenemos que avanzar con ellos, entonces las TIC's [sic] nos ayudan en eso (E16).

Yo creo que son muy importantes, pero primero que todo hay que saber utilizarlas y darle un buen uso, debería haber una capacitación previa al usar las TIC. Porque o sino la mal usan los papás o la educadora, en muchos casos para que el niño esté tranquilo le ponen un computador y sería, mejor debería existir una capacitación para aprender a usar las TIC y sacar provecho (E10). 


\subsection{Valoración de las TIC en su vida estudiantil}

En cuanto a la valoración que le atribuyen a las TIC en su vida estudiantil, cabe mencionar que los resultados se presentan con base en la percepción que poseen, desde tres perspectivas: i) Uso de estas herramientas desde la docencia, ii) Aporte de la asignatura denominada Tecnología y Aprendizaje (cursada en el primer año de la carrera). Finalmente, y con base en lo anterior, a partir de lo señalado por las estudiantes, se presentan algunas necesidades de formación en esta línea.

\subsubsection{Uso de estas herramientas desde la docencia}

Se observa una valoración positiva al respecto y se destaca que algunas personas docentes intentan utilizarlas para apoyar el proceso de enseñanza y aprendizaje más allá del uso de programas de presentaciones habituales, pero al mismo tiempo, hacen alusión a docentes que no las incorporan en sus clases, lo que para ellas también resulta ser una acción valorada, por cuanto indican que logran una mayor atención hacia quien enseña, con solo escuchar. Esta percepción puede ser evidenciada cuando exponen lo siguiente:

Algunas veces vamos al laboratorio, es que en algunas asignaturas vamos al laboratorio a hacer trabajos (E1).

A veces decían vamos a realizar un cuento, el libro, picto-libro, pero se hacía en el laboratorio y ahí la profesora decía al principio que eso se iba hacer allá, pero no estaba escrito en el programa (E3).

Es que a mí en lo personal me pasa que con las presentaciones de los profesores me distraigo porque estoy escribiendo algo de la presentación, pero el profesor o la profesora está hablando otra cosa entonces no sé si escribir o escuchar (E6).

Yo recuerdo que con un profe, nosotras nos sentábamos en la primera fila, porque como él no hacía presentaciones[...] al final lo que iba diciendo a nosotras igual nos quedaba súper claro, tomábamos apuntes y después nos iba súper bien, porque le poníamos atención a lo que él decía (E5).

El profesor no utilizaba presentaciones, él solamente hablaba y escribía en la pizarra , pero era lo mínimo, entonces nosotras [...] yo por lo menos estaba totalmente 
concentrada en lo que él estaba diciendo, en ese sentido, yo siento que su metodología estaba bien al no utilizar presentación (E2).

\subsubsection{Aporte de la asignatura de Tecnología y Aprendizaje}

Se observa una valoración bastante positiva hacia la asignatura de Tecnología y Aprendizaje, pues reconocen que fue provechosa porque lograron aprender herramientas digitales distintas a las tradicionales, las que además pudieron utilizar en otras asignaturas. No obstante, también se reconoce que no le otorgaron mayor importancia a lo que vieron en esta asignatura, por lo que el conocimiento logrado sobre el uso de algunas herramientas digitales solo tuvo sentido en la medida en que se constituían en un requisito de aprobación, vinculado a la evaluación, pero que al no continuar aplicándolas las llevó a olvidar lo aprendido:

Nos entregaban formación para trabajar la tecnología con el Prezzi, Cmaptool y otras, yo no conocía ninguna, las encuentro fantásticas y sirve como para ir cambiando. A mí me sirvió para las presentaciones en las otras asignaturas de la carrera, por eso es muy importante el tema del aprendizaje autónomo porque ella me enseñaba [...] yo tenía que seguir, entonces me gustaba, me sirvió mucho el primer año (E11).

El curso de TIC en general es de gran ayuda para la mayoría, descubrimos todo estos software y todas estas páginas que nos ayudaron a desarrollar muchos trabajos, Prezzi que fue una nueva forma de poder entregar la información que nosotras teníamos (E2).

El curso en sí es bueno, pero el tema está en que nosotras no le damos el valor que realmente deberíamos darle al curso, porque enseñan cosas muy importantes [...] planillas de cálculo sobre todo, que nos enseñaba como hacer las listas, no sé...contar en grandes cantidades, sacar promedios. Yo por lo menos no le di el valor que le debí dar a ese curso, porque también nos enseñaban a hacer cuentos, como hacer muchas cosas, pero en realidad yo ahora en estos momentos de muy poco me acuerdo, si veo las páginas si me acuerdo, pero yo siento que lo por lo menos no le di el valor que debí darle al curso de TIC, porque es muy bueno (E3). 
A partir de lo señalado por las futuras educadoras, se puede apreciar también que aún persisten necesidades de profundización en algunos softwares, particularmente en lo referido el uso, operación y manipulación de planillas de cálculo, pues manifiestan que poseen escasos conocimientos sobre su utilización.

Se destaca que la ubicación curricular de la asignatura Tecnología y Aprendizaje también se constituye en un aspecto que debería ser replanteado, pues al estar ubicada al inicio de su proceso formativo genera que, en los años siguientes, lo aprendido en ese momento se les olvide, sobre todo cuando no son incorporadas en el resto de las asignaturas. Del mismo modo, señalan que en determinados periodos de su formación requieren de hacer uso de ciertas herramientas tecnológicas y digitales para las que no han sido preparadas, es así como exponen lo siguiente:

Falta profundizamos en planillas de cálculo, porque tenemos como lo básico, pero creo que si se profundizara esa aplicación junto con la creación de páginas web o de blog seria de mucha gran ayuda (E8).

Con respecto a lo que es planillas de cálculo deberíamos tener un refuerzo, y estaba pensando que más a futuro la carrera podría plantearse no dar TIC en primer semestre porque, va en primer año y lo que más utilizamos es procesadores de texto y software de presentaciones, pero al llegar a tercero nos vemos enfrentadas a que tenemos que hacer más cosas, investigaciones, más planificaciones y ahí podríamos tener el curso para trabajar a la par con lo que vamos haciendo y mejorar el uso de las tecnologías (E12).

\subsection{Desempeño en el uso de TIC}

Esta categoría permitió un acercamiento a la autovaloración que las estudiantes le atribuyen a su nivel de conocimientos y/o desempeños en el uso de las herramientas tecnológicas y digitales, tanto en lo referido a su uso instrumental como desde el punto de vista pedagógico. Al respecto, se destaca que, en general, sienten poseer un nivel de conocimientos más bien bajo y, aunque tienen dominio de softwares tradicionales, como un procesador de texto o software de presentación, consideran que no saben darles un uso pedagógico:

Encuentro que todavía estoy en debe con aprender a utilizar correctamente las TIC porque aprender a utilizar no solamente saber cómo utilizar, sino que saberlas 
utilizarlas bien, darle un buen uso a las tecnologías que se nos presenta ya que a nosotros siempre nos enseñaron hacer una presentación, ya yo lo sé hacer puedo manejar perfectamente la parte de la como hacer una, pero cómo lo utilizo bien, cómo logro sacarle provecho en la función que representa la presentación en una clase es lo que me falta. Porque yo puedo ocupar lo que el programa dicta, pero ocuparla también es darle un buen uso (E10).

El software de presentación nos permite mostrar imágenes, pero también nos permite reproducir sonido y vídeos y nosotras es algo que no ocupamos mucho, como decía acá mi compañera, de presentar un video con las estaciones del año, vamos a poner solamente imágenes y en ningún momento se nos pudiera ocurrir que en el invierno e insertar el sonido de la lluvia en la misma presentación que estamos mostrando, eso nos dice que nosotros no sabemos utilizar, pero no le damos un uso correcto, no potenciamos el uso que tienen estas TIC (E12).

El procesador de texto puede...es una herramienta cotidiana, sobre todo los que estamos en la universidad porque todo trabajo que tenemos que entregar personalmente en mi grupo, si hay que hacer una presentación ponemos toda la información en un texto y después la clasificamos y la ordenamos las dispositivas, pero lo primero lo esencial es el texto (E8).

Publisher nos permite entregar información a los apoderados haciendo trípticos, dípticos, írselos entregando para que ellos manejen la información en cuanto a salud, juego, lecturas que puedan dar, igual con eso ellos se pueden informar (E2).

En este sentido, es importante destacar que las estudiantes lograron conocimientos en torno al uso de TIC antes de ingresar a la universidad, sin embargo, reconocen que es en su proceso formativo como educadoras donde han logrado alcanzar mayor nivel de profundidad en ellos, aunque fundamentalmente asociados al uso de un procesador de texto y software de presentación:

Teníamos un ramo que se llamaba computación y nos enseñan a usar un procesador de texto, accesorios, a hacer presentaciones y a usar una planilla de cálculo, pero acá los profundicé más (E1). 
Si bien las conocí en la enseñanza básica y media, yo las aprendí a usar en la universidad, pero es porque yo vengo de una carrera previa, entonces ahí yo aprendí en base a práctica y error, cómo se tenían que utilizar los programas, cómo hacer que las letras se vieran para todos, que los fondos no molestaran a los compañeros que estaban en el último lugar de la sala, eso yo lo aprendí en la U (E4).

\section{Discusión y conclusiones}

No se puede desconocer que uno de los actuales desafíos a los que se ve enfrentado el profesorado tiene relación con la implementación de las Tecnologías de la Información y la Comunicación dentro de su acción docente, desde una dimensión pedagógica, lo que supone un saber asociado a la capacidad para "aplicar las TIC en el currículo escolar vigente como una forma de apoyar y expandir el aprendizaje y la enseñanza" (MINEDUC, 2008). En este sentido, se puede señalar que la decisión de incorporar las tecnologías, como recurso para apoyar el proceso educativo, implica que la persona docente vaya más allá de saber darles un uso instrumental, pues requiere que sea capaz de reflexionar críticamente en torno a los aspectos epistemológicos que le dan sustento a sus prácticas pedagógicas y los vincule con el uso de estas herramientas en su práctica docente.

No se trata de integrar las TIC porque son recursos de moda o como una exigencia presentada desde las políticas educativas, sino que involucra situarse desde una forma distinta de comprender el proceso educativo (de la Herrán y Fortunato, 2017). De esta manera, se hace necesario recordar que el solo hecho de incorporar estas herramientas en la docencia no necesariamente permite afirmar que se esté aprovechando todo el potencial educativo que aportan (Flores-Lueg, 2017), o que por sí solas van a contribuir al mejoramiento de los aprendizajes.

En relación con lo anterior, consideramos importante precisar que la incorporación de las TIC en el aula no necesariamente conlleva a visualizar prácticas pedagógicas innovadoras que tienen un efecto positivo en el mejoramiento de los aprendizajes (Peirano y Domínguez, 2008; Ponce, López, Labra y Toro, 2012). Las razones de ello pueden ser diversas, algunas externas al profesorado y otras de carácter personal, tales como la actitud frente a los computadores e Internet, el nivel de conocimientos autopercibido en relación con el uso de estas herramientas, el género, el nivel educativo de formación (Flores-Lueg, 2015), entre otros factores. Por ello, sostenemos que la formación inicial docente se constituye en un momento clave para promover el desarrollo de aquellas competencias vinculadas al uso 
de las TIC en el proceso de enseñanza y aprendizaje, tanto de las futuras personas educadoras como la de los y las estudiantes que asisten a establecimientos del sistema educativo.

En lo que respeta al objetivo de este estudio, centrado en determinar el significado que estudiantes de la carrera de Pedagogía en Educación Parvularia le atribuyen a las TIC incorporadas en su proceso formativo desde una dimensión pedagógica, se puede evidenciar que las futuras educadoras han ido construyendo un significado sobre estas herramientas que responde a dos espacios diferentes. Un espacio referido al hogar, donde, a juicio de ellas, los párvulos entran en contacto con los objetos tecnológicos solamente con fines de entretención (efecto "babysitter"), lo que les privaría de oportunidades de aprendizajes logrados por medio de la exploración y, al mismo tiempo, afectaría en el proceso de socialización. El otro espacio, vinculado a la educación formal, donde se observa una significación diferente, pues las estudiantes destacan la posibilidad que ofrecen estas herramientas para que sean aprovechadas didácticamente en el proceso de enseñanza y aprendizaje de los párvulos. Argumentan que resultan ser más motivadoras y atractivas para ellos, pero que es necesario, previamente, tener una formación que les permita aprender a otorgarle un sentido pedagógico.

En cuanto a su formación en el uso de TIC, si bien perciben tener buen nivel de desempeño, dicha valoración se vincula principalmente al uso de softwares tradicionales, empleados con frecuencia en las distintas asignaturas de su trayecto formativo, entre ellos, el uso de un procesador de texto en particular y de un software de presentaciones. Aunque también se destaca que han tenido la oportunidad de utilizar algunos softwares que van más allá de los tradicionales, estos solo se han empleado en forma aislada, y el conocimiento adquirido sobre su utilización solo estuvo condicionado a la aprobación de una asignatura en particular (Vaillant, 2014), pues las escasas posibilidades de emplearlas o transferirlas a otros cursos, a lo largo del proceso formativo, ocasionó que se les olvidara lo que habían aprendido. Consecuentemente, se puede desprender que el desarrollo de habilidades vinculadas al manejo de herramientas tecnológicas requiere de situaciones que demanden de su utilización constante por parte de las estudiantes, pues no bastaría con que hagan uso de un software o cierta aplicación digital en un solo momento, pues ello no tendría un efecto duradero en el aprendizaje.

Por consiguiente, se considera necesario enfatizar que la incorporación de las TIC en el proceso de formación inicial del profesorado debe ser un aspecto abordado de manera 
transversal durante toda la etapa formativa, donde se les ofrezcan experiencias de carácter práctica, que permitan no solo aprender a usarlas en beneficio personal, sino, además, apropiarse de ellas visualizándolas como medios que pueden ser aprovechados para apoyar el proceso de enseñanza y aprendizaje, y con ello implementar estrategias didácticas contextualizadas y pertinentes a la realidad que viven las personas que asisten a la educación formal.

Por otro lado, es importante tener en consideración que el uso de las TIC, desde una dimensión pedagógica, requiere no solo de una preparación por parte de las estudiantes, sino que también se necesita de docentes que las valoren como recursos que pueden ser intencionados para favorecer procesos de aprendizaje más pertinentes a las exigencias que el contexto socioeducativo está demandando. En este sentido, sostenemos que es necesario enfatizar que la incorporación de estas herramientas en el aula, bajo la persistencia de un modelo educativo academista o tradicional, arraigado firmemente en las prácticas docentes, se constituye en uno de los factores clave que favorece la adquisición de conocimientos básicos e instrumentales sobre su utilización por parte del estudiantado, lo que tendría incidencia en el escaso conocimiento manifestado por las futuras educadoras respecto a cómo aplicar estas herramientas desde una perspectiva pedagógica (Flores-Lueg, 2015).

Finalmente, concluimos que sigue siendo relevante que se promueva una actitud favorable hacia el uso de las TIC, desde una dimensión pedagógica, a lo largo del proceso de formación inicial y que, al mismo tiempo, se fomenten espacios de reflexión crítica respecto del aporte que ofrecen estas herramientas para apoyar el proceso educativo de los párvulos.

Sumado a lo anterior, se considera necesario revisar los planes de formación de las carreras de pedagogía, bajo una mirada integradora, donde la implementación de las TIC adopte un carácter transversal en el itinerario formativo, con el fin de erradicar la lógica asignaturista o fragmentada bajo la cual se ha orientado la formación inicial del profesorado en este ámbito, pues una asignatura por sí misma no contribuye al desarrollo de aquellas competencias que habiliten a las futuras educadoras de párvulos a aprovechar todo el aporte que estas herramientas ofrecen desde el punto de vista pedagógico.

Se sugiere continuar desarrollando esta línea de investigación, teniendo en consideración el significado que construyen educadoras de párvulos que se encuentran desempeñando su labor en distintas realidades escolares de la región y del país, pues el acercamiento a estas significaciones puede arrojar información relevante que tribute a la 
transformación de los procesos formativos que ocurren en la formación inicial respecto a la apropiación de las TIC desde una perspectiva pedagógica. Si bien, este estudio se centró en una realidad particular, y por tanto, no admite generalizaciones, creemos importante también observar lo que ocurre con la incorporación de las TIC en otras instituciones de educación superior encargadas de formar a futuras educadoras de párvulos, tanto a nivel del diseño curricular como de su operacionalización, pues ello podría proporcionar otros insumos importantes a los centros formadores para tomar decisiones orientadas hacia la optimización de la formación inicial de educadoras en uso pedagógico de TIC.

\section{Referencias}

Abarzúa, Alejandra y Cerda, Cristian. (2011). Integración curricular de TIC en educación parvularia. Revista de Pedagogía, 32(90), 13-43. Recuperado de http://www.redalyc.org/articulo.oa?id=65920055002

Barrantes, Gerardo, Casas, Luis Manuel y Luengo, Ricardo. (2014). Competencias Tecnológicas de los profesores de Infantil y Primaria de Extremadura en función del género. Revista Iberoamericana de Informática Educativa, 19, 38-48. Recuperado de https://dialnet.unirioja.es/servlet/articulo?codigo=4794548

Bisquerra, Rafael (coord.). (2014). Metodología de la Investigación Educativa. 4ª Edición. Madrid: Editorial La Muralla.

Bravo, Astrid y Morales, Hernán. (2012). Caracterización de educadoras de párvulos que se desempeñan en el nivel sala cuna en una muestra de jardines infantiles de Concepción, Chile. Revista Electrónica Actualidades Investigativas en Educación, 12(2), 125. Recuperado de http://www.redalyc.org/pdf/447/44723437009.pdf

Cabero, Julio, Marín, Verónica y Castaño, Carlos Manuel. (2015). Validación del modelo TPACK para la formación del profesorado en TIC. @tic. Revista d`innovació educativa, (14), 13-22. Recuperado de http://roderic.uv.es/handle/10550/44762

Cascales, Antonia, Carrillo, María Encarnación y Redondo, Ana María. (2017). ABP y Tecnología en Educación Infantil. Pixel Bit, Revista de Medios y Educación, 50, 201209. doi: 10.12795/pixelbit.2017.i50.14

de la Herrán, Agustín y Fortunato, Iván. (2017). La clave de la educación no está en las nuevas Tecnologías de la Información y la Comunicación (TIC). Acta Scientiarum. Education, $39(3), \quad 311-317 . \quad$ Recuperado de http://www.redalyc.org/pdf/3033/303351030008.pdf

de Saint Pierre, Didier. (2008). Futuros profesores: ¿por qué formar en competencias digitales? Enlaces. Ministerio de Educación. Chile. Recuperado de http://enlacesenred.blogspot.com.es/2008/10/futuros-profesores-por-qu-formar-en.html 
Espinoza, Victoria y Rosas, Ricardo. (2016). Creencias de educadoras y miembros de equipos directivos de centros educativos de educación parvularia respecto del uso de recursos tecnológicos como herramientas de aprendizaje. Estudios pedagógicos (Valdivia), 42(2), 95-112. Doi http://dx.doi.org/10.4067/S0718-07052016000200006

Fainholc, Beatriz, Navia, Hugo, Romero, Rosita y Halal, Carol. (2013). La formación del profesorado y el uso pedagógico de las TIC. RED. Revista de Educación a Distancia, (38), 1-14. Recuperado de http://www.um.es/ead/red/38/fainholc.pdf

Flores-Lueg, Carolina. (2015). Factores que inciden en la valoración de estudiantes de Pedagogía sobre sus competencias digitales: el caso de la Universidad del BíoBío(Chile) (Tesis Doctoral). RUA. Repositorio Institucional Universidad de Alicante. Recuperado de http://hdl.handle.net/10045/50256

Flores-Lueg, Carolina and Roig-Vila, Rosabel. (2017). Gender and its impact on Pedagogy students' self-perceived digital competence. International Journal of Educational Research and Innovation (IJERI), (8), 79 -96.

Flores-Lueg, Carolina. (2017). Actitud de futuros maestros frente al uso de TIC en educación: un análisis descriptivo. Notandum, (44-45), 53-68. DOI: http://dx.doi.org/10.4025/notandum.44.6

García-Ruiz, Rosa, Duarte, Ana y Guerra, Sonsoles. (2014). Propuesta de un Instrumento de Evaluación para medir el grado de competencia mediática en la etapa de educación infantil. Píxel-Bit. Revista de Medios y Educación, (44), 81-96. doi: http://dx.doi.org/10.12795/pixelbit.2014.i44.06

Gértrudix, Felipe y Ballesteros, Verónica. (2014). Herramientas 2.0. Recursos innovadores integrados en los Proyectos Didácticos de Educación Infantil. Un estudio de caso. EDUTEC, Revista Electrónica de Tecnología Educativa, 48. Recuperado de http://www.edutec.es/revista/index.php/edutec-e/article/viewFile/60/20

González Pérez, Alicia y de Pablos, Juan. (2015). Factores que dificultan la integración de las TIC en las aulas. Revista de Investigación Educativa, 33(2), 401-417. doi: http://dx.doi.org/10.6018/rie.33.2.198161

Guillemette, François. (2006). L'approche de la Grounded Theory; pour innover? RECHERCHES QUALITATIVES, 26(1), 32-50. Recuperado de https://goo.gl/90NeHw

Gutiérrez Martín, Alfonso. (2012). Formación del profesorado para la alfabetización múltiple. En Rosa María Sáinz Peña (coord.), Alfabetización digital y competencias informacionales (pp.43-98). Barcelona: Editorial Aljibe y Fundación Telefónica.

Gutiérrez, Isabel and Serrano, José Luis. (2016). Evaluation and development of digital competence in future primary school teachers at the University of Murcia. Journal of New Approaches in Educational Research, 5(1), 51-56. 
Hervás-Gómez, Carlos y Silva-Carmona, María del Carmen. (2016). Las TIC en el ámbito de la educación infantil en la provincia de Sevilla. En José Gómez-Galán, Eloy LópezMeneses y Laura Molina-García (Eds.), Instructional Strategies in Teacher Training (pp.31-36). UMET Press, Universidad Metropolitana, Sistema Universitario Ana G. Méndez, San Juan, PR

Kale, Ugur and Goh, Debbie. (2014). Teaching style, ICT experience and teachers' attitudes toward teaching with Web 2.0. Educ Inf Technol, 19(1), 41-60. doi: 10.1007/s10639012-9210-3

Katz, Lilian. (2005). Perspectivas educativas en la primera infancia. Santiago de Chile: Ediciones Lom.

Marín-Barbero, Jesús. (2009). Cuando la tecnología deja de ser una ayuda didáctica para convertirse en una mediación cultural. Revista Electrónica de Teoría de la Educación. Educación y Cultura en la Sociedad de la Información, 10(1), 19-31. Recuperado de https://goo.gl/VGHPlc

Marín-Díaz, Verónica y Martín-Párraga, Javier. (2014). ¿Podemos utilizar los videojuegos para el desarrollo del currículo de la etapa de infantil? New Approaches in Educational Research, 3(1), 21-27.

Ministerio de Educación de Chile (MINEDUC). (2001). Bases Curriculares de la Educación Parvularia. Santiago: Gobierno de Chile

Ministerio de Educación de Chile (MINEDUC). (2008). Estándares TIC para la Formación Inicial Docente. Una propuesta para el contexto chileno. Santiago de Chile: Red Enlaces/ UNESCO. Recuperado de http://unesdoc.unesco.org/images/0016/001631/163149s.pdf

Ministerio de Educación de Chile (MINEDUC). (2014). Estado del Arte de la Educación Parvularia en Chile. Santiago de Chile: Centro de Estudios MINEDUC.

Ministerio de Educación de Chile (MINEDUC). (2015). Resolución Exenta 2923. Aprueba nómina de establecimientos del "Proyecto Tablet para Educación Inicial en NT1, NT2 y $1^{\circ}$ Básico" en el marco del Programa de Informática Educativa en Escuelas y Liceos Enlaces. Recuperado de http://www.enlaces.cl/conoce-los-establecimientosseleccionados-en-proyectos-2016-tablet-para-ed-inicial-y-estandar-de-equipamientoen-ceia-modelos/

Peirano, Claudia y Domínguez, María Paz. (2008). Competencia en TIC. El mayor desafío para la evaluación y el entrenamiento docente en Chile. Revista Iberoamericana de Evaluación Educativa, 1(2), 106-123. Recuperado de http://www.rinace.net/riee/numeros/vol1-num2/art7.pdf

Pérez, Francisco y Vílchez, José Eduardo. (2013). Percepción de futuros maestros sobre el potencial de las TIC en la educación: de las expectativas a la realidad. Revista Fuentes, 13, 155-172. Recuperado de http://institucional.us.es/revistas/fuente/13/campo abierto 3.pdf 
Ponce, Héctor, López, Mario, Labra, Juan y Toro, Oscar. (2012). Integración curricular de organizadores gráficos interactivos en la formación de profesores. Revista de Educación, (357), 397-422. doi: 10.4438/1988592X-RE-2010-357-066

Quintanal, José y García, Begoña (coord.). (2012). Fundamentos básicos de metodología de investigación educativa. Madrid: Editorial CCS.

Ramírez-Abrahams, Patricia, Patiño-Mora, Vivian y Gamboa-Vásquez, Elsa. (2014). La educación temprana para niños y niñas desde el nacimiento a los 3 años: Tres perspectivas de análisis. Revista Electrónica Educare, 18(3), 67-90. doi: http://dx.doi.org/10.15359/ree.18-3.5

Sáinz, Milagros. (2013). El uso de las TIC en el ámbito educativo con perspectiva de género. Actitudes del profesorado y del alumnado. TELOS (Cuadernos de Comunicación e Innovación), (95), 116-124. Recuperado de https://goo.gl/g0aVHP

San Martín, Daniel. (2014). Teoría fundamentada y Atlas.ti: recursos metodológicos para la investigación educativa. REDIE. Revista Electrónica de Investigación Educativa,16(1), 104-122. Recuperado de http://www.redalyc.org/pdf/155/15530561008.pdf

Silva, Juan y Astudillo, Andrea. (2012). Inserción de TIC en la formación inicial docente: barreras y oportunidades. Revista Iberoamericana de Educación, 58(4), 1-11. Organización de Estados Iberoamericanos para la Educación, Ciencia y la Cultura (OEI). Recuperado de http://www.rieoei.org/deloslectores/4557Silva.pdf

Silva, Juan. (2012). Estándares TIC para la Formación Inicial Docente: una política pública en el contexto chileno. Archivos Analíticos de Políticas Educativas, 20(7), 1-40. Recuperado de http://epaa.asu.edu/ojs/article/viewFile/962/963

Suárez-Rodríguez, Jesús, Almerich, Gonzalo, Díaz-García, Isabel y Fernández-Piqueras, Rocío. (2012). Competencias del profesorado en las TIC. Influencia de factores personales y contextuales. Universitas Psychologica, 11(1), 293-309. Recuperado de http://revistas.javeriana.edu.co/index.php/revPsycho/article/viewFile/997/1537

Touriñán, José Manuel y Sáez, Rafael. (2012). Teoría de la educación, metodología y focalizaciones. La mirada pedagógica. La Coruña: Netbiblo.

Unesco. (2004). Las Tecnologías de la Información y la Comunicación en la Formación Docente. Guía de Planificación. Uruguay: Ediciones TRILCE. Recuperado de http://unesdoc.unesco.org/images/0012/001295/129533s.pdf

Vaillant, Denise. (2014). Formación de profesores en escenarios TIC. Revista e-Curriculum, São Paulo, 2(12), 1128-1142. Recuperado de http://www.redalyc.org/html/766/76632206003/

Vaillant, Denise. (2013). Integración de TIC en los sistemas de formación docente inicial y continua para la Educación Básica en América Latina. Argentina: Fondo de las Naciones Unidas para la Infancia (UNICEF). Recuperado de http://www.unicef.org/argentina/spanish/educacion Integracion TIC sistemas formaci on docente.pdf 